\title{
Equilíbrio ácido-base e hidroeletrolítico em equinos submetidos à simulação de enduro FEI"
}

[Acid-base and hidroelectrolytic balance in horses undergoing $F E I *$ endurance ride simulation]

\author{
N.S. Bernardi ${ }^{1}$, L.M.W. Gomide ${ }^{1}$, A.D. Antunes ${ }^{1}$, K. Gravena ${ }^{1}$, D.P.M. Dias ${ }^{1}$, \\ D.J. Queiroz ${ }^{1}$, V.A. Canello ${ }^{1}$, A.M.G.B. Silva ${ }^{1}$, R.A. Brandi ${ }^{2}$, J.C. Lacerda-Neto ${ }^{1}$ \\ ${ }^{1}$ Universidade Estadual Paulista "Júlio de Mesquita Filho" - Unesp - Jaboticabal, SP \\ ${ }^{2}$ Universidade de São Paulo - USP - Pirassununga, SP
}

\begin{abstract}
RESUMO
Enduro é uma modalidade equestre que demanda alta exigência dos sistemas orgânicos para que seja mantida a homeostasia do organismo. Objetivou-se avaliar o equilíbrio ácido-base e hidroeletrolítico durante uma prova de enduro realizada por equinos FEI*. Nove equinos foram submetidos a três meses de treinamento e, ao final, realizou-se prova similar às competições oficiais de enduro equestre, com trajeto de $80 \mathrm{~km}$, dividido em quatro etapas (anéis). Avaliações clínicas e coletas de amostras sanguíneas para análise da concentração de lactato e variáveis hemogasométricas foram realizadas no início da prova, imediatamente após a chegada de cada anel e, após 15 e 30 minutos, uma, quatro, seis e 12 horas do término da prova. Apenas cinco animais concluíram a prova e, para análise estatística, foram utilizados somente os dados destes animais. Houve predomínio do metabolismo aeróbio durante a prova e os animais mostraram bom índice de recuperação. Os eletrólitos cloreto, sódio, potássio e cálcio tiveram sua concentração diminuída, enquanto o bicarbonato aumentou no transcorrer do exercício. Os valores de $\mathrm{pH}$ aumentaram em decorrência do desenvolvimento de alcalose mista, caracterizada por alcalose metabólica hipoclorêmica e alcalose respiratória. O bom desempenho dos animais foi atribuído à suficiente adaptação metabólica induzida pelo treinamento.
\end{abstract}

Palavras-chave: cavalo, eletrólitos, exercício, hemogasometria, lactato

\begin{abstract}
Endurance is an equestrian modality that demands high requirement of organic systems in order to maintain organism's homeostasis. This study aimed to evaluate hydroelectrolyte and acid-base balance during an endurance test performed by FEI* horses. Nine horses were subjected to three months of training and, at the end, performed a test similar to the official competitions of equestrian endurance, with $80 \mathrm{~km}$ of distance, divided into four stages. Clinical and blood samples were taken for analysis of lactate concentration and blood gas parameters before the start of the test, immediately after the arrival of each phase and, after 15, 30 minutes, 1, 4, 6 and 12 hours after the end of the race. Only five animals completed the race and the data from these animals were used for statistical analysis. There was predominance of aerobic metabolism and the horses showed a good recovery rate. The electrolytes chloride, sodium, potassium and calcium had their concentration decreased while bicarbonate increased with the course of the exercise. There was an increase in $\mathrm{pH}$ due to the development of mixed alkalosis, characterized by hypochloremic metabolic alkalosis and respiratory alkalosis. Good performance of the animals was attributed to sufficient metabolic adaptation induced by training.
\end{abstract}

Keywords: horse, electrolytes, exercise, blood gas analysis, lactate

Recebido em 29 de agosto de 2016

Aceito em 20 de abril de 2017

E-mail: nara.sb@gmail.com 


\section{INTRODUÇÃO}

O enduro equestre é a competição que visa testar a habilidade do atleta em administrar com segurança a resistência e o condicionamento de seu cavalo contra o trajeto, a distância, o clima, o terreno e o relógio (Endurance..., 2016). Essa modalidade é o segundo esporte equestre mais praticado no mundo, atrás apenas do salto. Atualmente o mais importante não é o cavalo ser o mais rápido, mas sim chegar em boas condições. A modalidade foi regulamentada pela Fédération Equestre Internationale (FEI) em 1982, e, a partir de então, todas as provas internacionais seguem as regras da federação (Nagy et al., 2012). As categorias são divididas de acordo com a distância percorrida, e a categoria Concours d'Endurance International* (CEI*) é aquela em que o cavalo e o cavaleiro devem percorrer entre 80 e $119 \mathrm{~km}$ em um único dia (Endurance..., 2016).

A competição é dividida em etapas (anéis) de cerca de $20-40 \mathrm{~km}$. Nas provas atuais, a maioria dos anéis são organizados em torno de uma área central (Vet Gate), de onde os cavalos começam e para onde retornam após cada ciclo. Antes do início da prova e após cada anel, os cavalos passam por inspeção veterinária, podendo ser eliminados se, no decorrer do exame, for constatado que o estado clínico ou ortopédico do animal não está adequado para continuar o percurso. Eliminações podem ocorrer unicamente devido à mensuração da frequência cardíaca acima do valor definido no cronograma (geralmente maior que 64 batimentos por minuto) (Nagy et al., 2012).

Diversos fatores atuam quebrando a homeostasia de um organismo, entre eles o frio, o calor, o jejum e, principalmente, o exercício. Entre esses fatores, destacam-se os distúrbios ácido-base e eletrolíticos associados a exercícios prolongados e provas de enduro, que já foram bem descritos em cavalos (Robert et al., 2010; Silva et al., 2009; Teixeira-Neto et al., 2004). A interpretação das alterações no equilíbrio ácidobase que ocorrem durante eventos esportivos é complicada porque as principais variáveis alteram-se simultaneamente e, em geral, em direções opostas, o que pode mascarar alguns distúrbios. Devido à natureza longa e exaustiva das provas de enduro, ocorrem elevadas perdas de eletrólitos pelo suor, visto que o suor equino é hipertônico em relação ao plasma (Schott e Hinchcliff, 1993).

Em equinos, após exercício máximo, diminuições no $\mathrm{pH}$ estão relacionadas, principalmente, aos metabólitos do metabolismo anaeróbio. Já o aumento no pH, comum em provas de enduro, está relacionado ao grande volume de suor eliminado, quando ocorrem grandes perdas de cloreto, o que leva ao desenvolvimento de alcalose metabólica hipoclorêmica (Constable, 1997; Lindinger, 2004).

O objetivo do presente estudo foi avaliar o equilíbrio ácido-base e hidroeletrolítico durante uma simulação de prova de enduro realizada por equinos FEI*, bem como a recuperação após a competição, com 0 intuito de fornecer informações úteis na prevenção e no tratamento dos possíveis distúrbios metabólicos e musculoesqueléticos que culminam com a eliminação dos animais das competições.

\section{MATERIAL E MÉTODOS}

O protocolo experimental realizado no presente estudo está de acordo com os princípios éticos adotados pelo Colégio Brasileiro de Experimentação Animal (Cobea) e foi aprovado pela Comissão de Ética e Bem-Estar Animal (Cebea) da Faculdade de Ciências Agrárias e Veterinárias / Unesp - Campus de Jaboticabal (Protocolo \#00162411).

Foram utilizados nove equinos da raça Puro Sangue Árabe, dois garanhões, cinco machos castrados e duas fêmeas, com idade média de $9 \pm 1$ anos. Os equinos foram considerados hígidos segundo exame físico geral e específico para avaliação do sistema locomotor.

O treinamento teve duração total de três meses, nos quais os equinos foram exercitados três vezes por semana em dias alternados. No primeiro mês, os animais realizaram duas sessões semanais de 60min de duração, com predomínio de passo à velocidade média de 5,5 a $6 \mathrm{~km} / \mathrm{h}$, determinada por sistema GPS (RCX5-G5TM PolarEquine - Heart Rate Monitor). O percurso foi realizado em trilhas de terra batida ou sobre gramíneas, na região montanhosa ao redor da fazenda. A terceira sessão semanal de atividade física compreendeu 30 minutos de galope sem 
interrupção, realizado em pista de areia plana, à velocidade média de 15 a $18 \mathrm{~km} / \mathrm{h}$, também determinada por sistema GPS. No segundo e no terceiro mês de treinamento, houve incrementos de 30 minutos em cada sessão de exercício.

Uma semana após o término do período de treinamento, realizou-se uma prova similar às competições oficiais de enduro equestre CEI*. Os equinos percorreram um trajeto de $80 \mathrm{~km}$ de distância, dividido em quatro etapas (anéis), de $30 \mathrm{~km}, 20 \mathrm{~km}, 15 \mathrm{~km}$ e $15 \mathrm{~km}$. Foram realizados intervalos de 50 minutos entre cada anel. Avaliações clínicas e coletas de amostras de sangue da veia jugular (avaliação hemogasométrica e determinação da concentração de lactato) foram realizadas antes do início do teste de resistência, imediatamente após o término de cada anel, e após 15 e 30 minutos e uma, quatro, seis e 12 horas do término da prova. Para a dosagem de cloreto e proteína total, os tempos foram os mesmos, exceto nos momentos $15 \mathrm{~min}$, 30min, quatro horas e seis horas após o exercício, em que não houve avaliação. Obtiveram-se $9 \mathrm{~mL}$ de sangue venoso por coleta, sendo $3 \mathrm{~mL}$ destinados à avaliação hemogasométrica, $5 \mathrm{~mL}$ para a dosagem da concentração sérica de íons cloreto [ $\left.\mathrm{Cl}^{-}\right]$e proteína total e $1 \mathrm{~mL}$ destinado à análise da concentração de lactato.

Utilizou-se um analisador de gases, eletrólitos e hemoglobina total (i-STAT analyzer, Cartucho CG8+), determinando-se, imediatamente após a coleta, as concentrações venosas de sódio $\left(\mathrm{Na}^{+}\right)$, potássio $\left(\mathrm{K}^{+}\right)$cálcio ionizado $\left(\mathrm{Ca}^{++}\right)$, bicarbonato $\left(\mathrm{HCO}_{3}{ }^{-}\right)$, pressão parcial do gás carbônico $\left(\mathrm{CO}_{2}\right)$, pressão parcial do oxigênio $\left(\mathrm{O}_{2}\right)$, pH sanguíneo $(\mathrm{pH})$, hemoglobina total $(\mathrm{Hb})$, saturação de oxigênio $\left(\mathrm{SO}_{2}\right)$, hematócrito $(\mathrm{Ht})$, excesso de base (EB) e glicose. Em todos os momentos de coleta sanguínea para determinação da hemogasometria, aferiu-se a temperatura retal por meio de termômetro digital ultrarrápido, já que algumas variáveis sofrem correção da temperatura.

Cinco mililitros de sangue foram imediatamente armazenados em tubos secos. As amostras foram centrifugadas durante 10 minutos a $1500 \mathrm{G}$, e o soro separado, congelado e armazenado à temperatura de $-20^{\circ} \mathrm{C}$ para posterior dosagem da $\left[\mathrm{Cl}^{-}\right]$( Cloretos Liquiform - Labtest Diagnóstica
SA) e de proteínas totais (Cloretos Liquiform Labtest Diagnóstica SA ).

Para determinação da concentração de lactato, adicionou-se $1 \mathrm{~mL}$ de sangue a $2 \mathrm{~mL}$ de fluoreto de sódio a $1 \%$ em tubos plásticos, à semelhança do que foi feito por Gomide et al. (2006) e Oliveira et al. (2006). Na sequência, os tubos foram inicialmente resfriados por imersão em gelo e posteriormente congelados a $-20^{\circ} \mathrm{C}$. A concentração de lactato de cada amostra foi determinada pelo método da lactato oxidase em analisador automático (YSI 2300 STAT Plus Glucose \& Lactate Analyzer).

Logo após as coletas sanguíneas, os animais foram resfriados e receberam água e feno à vontade. $\mathrm{O}$ exame físico foi realizado 20 minutos após a chegada de cada anel. Foram avaliadas ausência/presença de claudicação, frequência cardíaca (FC), frequência respiratória (FR), temperatura retal, perfusão tecidual (coloração de mucosas e tempo de preenchimento capilar), elasticidade cutânea e sensibilidade dos músculos lombares e pélvicos. O exame físico durante e ao término do enduro teve a finalidade de identificar quais animais estavam em condições de prosseguir o esforço; assim, alterações no andamento ou incapacidade em reduzir a FC no momento do exame abaixo de $64 \mathrm{bpm}$ foram itens de eliminação.

As variáveis estudadas passaram por teste de homocedasticidade pelo teste de Bartlett e foram avaliadas quanto à sua normalidade pelo teste de Shapiro-Wilk a $5 \%$ de significância. O delineamento utilizado foi inteiramente ao acaso, em que cada tempo representou um tratamento. As variáveis foram submetidas à análise de variância (ANOVA), e os valores médios comparados pelo teste de Tukey, com nível de significância $\mathrm{P}<0,05$, utilizando-se o software SAS (Statistical Analysis System).

\section{RESULTADOS}

O enduro ocorreu conforme proposto. Dos nove animais que iniciaram a prova, quatro foram eliminados ao decorrer do percurso. Portanto, os dados apresentados referem-se às variáveis analisadas nos cinco animais que terminaram o esforço de longa duração. No dia da realização do enduro, o índice pluviométrico foi de $14 \mathrm{~mm}$, o índice de umidade relativa do ar foi superior a 
90\% em todos os momentos e a temperatura apresentou média de $27^{\circ} \mathrm{C}$. Em decorrência das más condições climáticas que acabaram prejudicando muito a qualidade do solo, que se tornou enlameado por todo o percurso, a velocidade média dos animais ao longo da prova foi de $12,5 \mathrm{~km} / \mathrm{h}$, intercalando trote e cânter de acordo com as condições do terreno.

Os valores médios de hematócrito e hemoglobina apresentaram o mesmo padrão, aumentando com o transcorrer do exercício e retornando a valores basais quatro horas após o término dele (Tab. 1). $\mathrm{O}$ pH aumentou durante o enduro, porém retornou aos valores basais 15 minutos após o término do exercício. Excesso de base e bicarbonato também se elevaram durante o exercício, mas retornaram aos valores basais após uma hora de recuperação. A $\mathrm{pCO}_{2}$ aumentou após 15 e 30min do término do exercício, retornando aos valores basais após uma hora de recuperação. As concentrações de cloreto diminuíram ao fim do terceiro anel e em uma hora de recuperação, mas $12 \mathrm{~h}$ após o exercício apresentaram-se em concentração semelhante à basal (Fig. 1). Os valores de $\mathrm{pO}_{2}$ diminuíram após o término do exercício, retornando ao basal uma hora após o fim dele; a $\mathrm{SO}_{2}$ reduziu aos $15 \mathrm{~min}$, 30min e uma hora de recuperação, retornando aos valores basais após quatro horas do fim do exercício (Tab. 1). Já o lactato foi maior que os valores basais ao fim do primeiro anel e aos 15 e 30 minutos de recuperação, retornando aos valores iniciais após uma hora do fim do exercício (Tab. 1).

Tabela 1. Valores médios \pm desvio-padrão das variáveis sódio $(\mathrm{mmol} / \mathrm{L})$, potássio $(\mathrm{mmol} / \mathrm{L})$, cálcio ionizado $(\mathrm{mmol} / \mathrm{L})$, glicose $(\mathrm{mg} / \mathrm{dL})$, pressão de oxigênio $\left(\mathrm{pO}_{2}, \mathrm{mmHg}\right)$, saturação de oxigênio $\left(\mathrm{SO}_{2}, \%\right)$, hematócrito (Hct, \%), hemoglobina $(\mathrm{Hb}, \mathrm{g} / \mathrm{dL})$, excesso de base $(\mathrm{EB})$, temperatura retal $\left(\mathrm{TR},{ }^{\circ} \mathrm{C}\right)$ e de lactato (mmol/L) durante e após o exercício de longa duração

\begin{tabular}{|c|c|c|c|c|c|c|c|c|c|c|c|}
\hline & Avaliaçõ & ões & & & & & & & & & \\
\hline & Repouso & $01^{\circ} \mathrm{Anel}$ & $2^{\circ}$ Anel & $3^{\circ}$ Anel & $4^{\circ}$ Anel & $15 \mathrm{~min}$ & $30 \mathrm{~min}$ & $1 \mathrm{~h}$ & $4 \mathrm{~h}$ & $6 \mathrm{~h}$ & $12 \mathrm{~h}$ \\
\hline & 138,60 & 140,00 & 139,60 & 136,20 & 135,00 & 135,80 & 135,40 & 135,20 & 134,20 & 134,00 & 135 \\
\hline $\mathrm{Na}$ & $\pm 0,89^{\mathrm{ab}}$ & $\pm 2,00^{\mathrm{a}}$ & $\pm 3,58^{\mathrm{a}}$ & $\pm 2,17^{\mathrm{bcd}}$ & $\pm 1,58^{\mathrm{cd}}$ & $\pm 1,30^{\mathrm{bcd}}$ & $\pm 1,52^{\mathrm{cd}}$ & $\pm 1,79^{\mathrm{cd}}$ & $\pm 1,64^{\mathrm{d}}$ & $\pm 1,87^{\mathrm{d}}$ & $\pm 1,87^{\mathrm{cd}}$ \\
\hline & 3,74 & 3,08 & 2,90 & 2,92 & 2,88 & 2,82 & 2,46 & 2,18 & 2,68 & 2,32 & 2,42 \\
\hline K & $\pm 0,09^{\mathrm{a}}$ & $\pm 0,23^{\mathrm{b}}$ & $\pm 0,19^{\mathrm{bc}}$ & $\pm 0,23^{\mathrm{bc}}$ & $\pm 0,31^{\text {bcd }}$ & $\pm 0,27^{\mathrm{bc}}$ & ${ }^{e} \pm 0,13^{c c}$ & ${ }^{2}=0,35^{\mathrm{g}}$ & $\pm 0,45^{\text {bcdef }}$ & $\pm 0,43^{\mathrm{fg}}$ & $\pm 0,41^{\text {defg }}$ \\
\hline & 1,73 & 1,51 & 1,48 & 1,43 & 1,42 & 1,45 & 1,48 & 1,52 & 1,54 & 1,58 & 1,51 \\
\hline $\mathrm{Ca}$ & $\pm 0,02^{\mathrm{a}}$ & $\pm 0,11^{\mathrm{bc}}$ & $\pm 0,19^{c}$ & $\pm 0,12^{\mathrm{c}}$ & $\pm 0,11^{\mathrm{c}}$ & $\pm 0,11^{\mathrm{c}}$ & $\pm 0,08^{c}$ & $\pm 0,07^{\mathrm{bc}}$ & $\pm 0,05^{\mathrm{bc}}$ & $\pm 0,03^{\mathrm{abc}}$ & \pm 0 \\
\hline & $e^{123,40}$ & 132,40 & 114,00 & 112,40 & 118,00 & 115,80 & 111,20 & 116,80 & 113,60 & 126,00 & 113 \\
\hline & $\pm 17,13^{\mathrm{a}}$ & $\pm 9,86^{\mathrm{a}}$ & $\pm 20,66^{\circ}$ & $\pm 21,15^{\mathrm{a}}$ & $\pm 25,05^{\mathrm{a}}$ & $\pm 20,71^{\mathrm{a}}$ & $\pm 19,99^{\mathrm{a}}$ & $\pm 19,25^{\mathrm{a}}$ & $\pm 16,86^{\mathrm{a}}$ & $\pm 9,22^{\mathrm{a}}$ & $\pm 9,85^{\mathrm{a}}$ \\
\hline & 38,80 & 45,60 & 45,20 & 41,40 & 43,40 & 23,20 & 25,40 & 29,60 & 31,00 & 30,00 & 29,8 \\
\hline $\mathrm{pO}_{2}$ & $\pm 5,45^{\mathrm{abc}}$ & $\pm 7,44^{\mathrm{a}}$ & $\pm 4,66^{\mathrm{a}}$ & $\pm 2,61^{\mathrm{ab}}$ & $\pm 6,77^{\mathrm{a}}$ & $\pm 7,69^{\mathrm{e}}$ & $\pm 9,37^{\mathrm{de}}$ & $\pm 5,86^{\text {cde }}$ & $\pm 4,30^{\text {cde }}$ & $\pm 2,65^{\text {cde }}$ & $\pm 3,70^{\text {cde }}$ \\
\hline & 72,40 & 79,20 & 79,80 & 76,60 & 76,60 & 36,60 & 41,40 & 49,20 & 60,20 & 59,40 & 61,4 \\
\hline $\mathrm{SU}_{2}$ & $\pm 7,09^{\mathrm{abc}}$ & $\pm 9,18^{\mathrm{a}}$ & $\pm 3,27^{\mathrm{a}}$ & $\pm 4,39^{\mathrm{ab}}$ & $\pm 8,20^{\mathrm{ab}}$ & $\pm 19,03^{\mathrm{f}}$ & $\pm 22,53^{\text {ef }}$ & $\pm 16,81^{\mathrm{dc}}$ & $\pm 8,17^{\mathrm{abc}}$ & $\pm 5,03^{\text {abcde }}$ & $\pm 6,35^{\text {abcdcdee }}$ \\
\hline & 39,40 & 47,20 & 49,00 & 47,00 & 47,60 & 47,20 & 50,00 & 47,00 & 44,00 & 43,40 & 42,40 \\
\hline Het & $\pm 5,86^{\mathrm{d}}$ & $\pm 6,06^{\mathrm{abc}}$ & $\pm 5,24^{\mathrm{a}}$ & $\pm 6,04^{\mathrm{abc}}$ & $\pm 6,27^{\mathrm{abc}}$ & $\pm 5,40^{\mathrm{abc}}$ & $\pm 4,30^{\mathrm{a}}$ & $\pm 4,30^{\mathrm{abc}}$ & $\pm 7,31^{\text {abcd }}$ & $\pm 6,35^{\mathrm{bcd}}$ & $\pm 6,02^{\mathrm{bcd}}$ \\
\hline & 13,40 & 16,06 & 16,66 & 15,98 & 16,20 & 16,04 & 17,00 & 16,00 & 14,98 & 14,76 & 14,40 \\
\hline $\mathrm{Hb}$ & $\pm 1,97^{\mathrm{d}}$ & $\pm 2,04^{\mathrm{abc}}$ & $\pm 1,78^{\mathrm{ab}}$ & $\pm 2,07^{\mathrm{abc}}$ & $\pm 2,16^{\mathrm{abc}}$ & $\pm 1,84^{\mathrm{abc}}$ & $\pm 1,45^{\mathrm{a}}$ & $\pm 1,48^{\mathrm{abc}}$ & $\pm 2,49^{\mathrm{abcd}}$ & $\pm 2,17^{\text {bcd }}$ & $\pm 2,05^{\mathrm{b}}$ \\
\hline & 8,00 & 11,20 & 12,60 & 13,40 & 14,20 & 14,80 & 13,60 & 11,40 & 10,80 & 10,20 & 11,8 \\
\hline EB & $\pm 1,73^{\mathrm{c}}$ & $\pm 2,59^{\mathrm{abc}}$ & $\pm 1,95^{\mathrm{a}}$ & ${ }^{\mathrm{c}} \pm 2,30^{\mathrm{ab}}$ & $\pm 2,59^{\mathrm{ab}}$ & $\pm 3,11^{\mathrm{a}}$ & $\pm 3,65^{\mathrm{ab}}$ & $\pm 1,52^{\mathrm{abc}}$ & $\pm 2,59^{\mathrm{abc}}$ & $\pm 4,38^{\mathrm{abc}}$ & $\pm 4,02^{\mathrm{abc}}$ \\
\hline & 37,48 & 39,04 & 39,44 & 38,90 & 39,10 & 38,78 & 38,72 & 38,38 & 37,76 & 37,32 & 36,74 \\
\hline $\mathrm{TR}$ & $\pm 0,30^{\mathrm{de}}$ & $\pm 0,36^{\mathrm{ab}}$ & $\pm 0,15^{\mathrm{a}}$ & $\pm 0,45^{\mathrm{ab}}$ & $\pm 0,39^{\mathrm{ab}}$ & $\pm 0,11^{\mathrm{ab}}$ & $\pm 0,18^{\mathrm{ab}}$ & $\pm 0,36^{\mathrm{bc}}$ & $\pm 0,29^{c}$ & $\pm 0,26^{\mathrm{de}}$ & $\pm 0,17$ \\
\hline Lacta & 0,68 & 1,25 & 1,87 & 1,17 & 1,13 & 1,68 & 1,71 & 1,4 & 0,88 & 0,88 & 0,71 \\
\hline & $\pm 0,25^{\mathrm{e}}$ & $\pm 0,42^{\mathrm{abc}}$ & $\pm 0,84^{\mathrm{a}}$ & $\pm 0,44^{\mathrm{ab}}$ & $\pm 0,45^{\mathrm{ab}}$ & $\pm 0,72^{\mathrm{abc}}$ & $\pm 0,3^{\mathrm{ab}}$ & $\pm 0,49^{\mathrm{abcc}}$ & ${ }^{\mathrm{de}} \pm 0,21^{\mathrm{cde}}$ & $\pm 0,19^{\text {cde }}$ & $\pm 0,18^{\mathrm{de}}$ \\
\hline
\end{tabular}

Letras diferentes na mesma linha indicam diferença pelo teste de Tukey $(\mathrm{P}<0,05)$. 

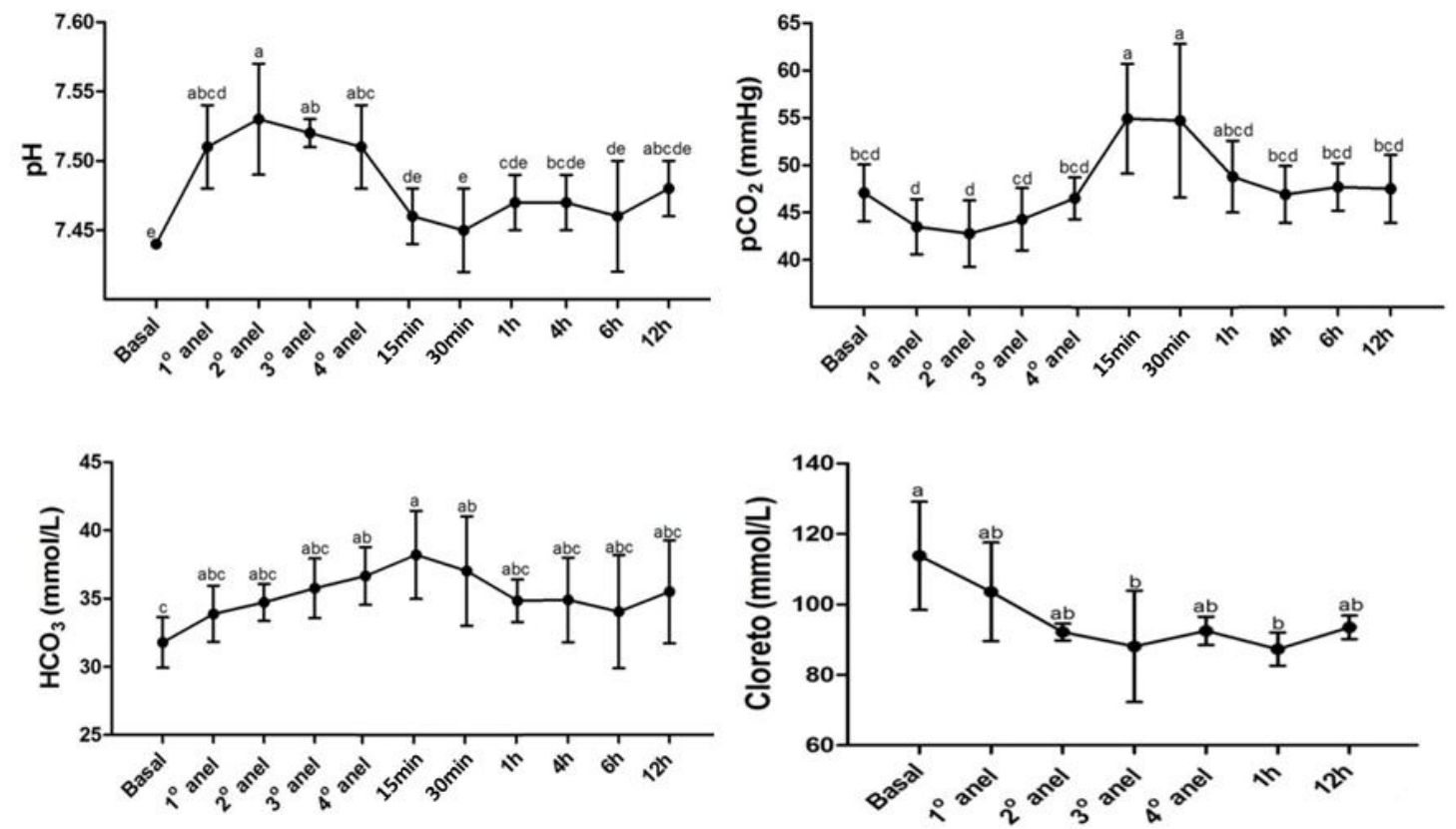

Figura 1. Variação dos valores médios \pm desvio-padrão do $\mathrm{pH}$, bicarbonato $(\mathrm{mmol} / \mathrm{L}), \mathrm{pCO} 2(\mathrm{mmHg}) \mathrm{e}$ cloreto $(\mathrm{mmol} / \mathrm{L})$ durante e após a realização de um enduro de $80 \mathrm{~km}$. Médias seguidas de letras diferentes divergem pelo teste de Tukey $(\mathrm{P}<0,05)$.

O sódio diminuiu após o último anel, retornou aos valores basais aos $15 \mathrm{~min}$, mas depois reduziu a sua concentração novamente e, após transcorridas 12 horas do término do exercício, ainda não havia retornado aos valores da concentração basal. Os eletrólitos potássio e cálcio tiveram sua concentração diminuída com o transcorrer do exercício, que também não retornaram aos valores basais após o término dele. A glicemia manteve-se constante ao longo do exercício e nos momentos de recuperação (Tab. 1). A proteína total não apresentou alteração significativa (Tab. 2).

Tabela 2. Valores médios \pm desvio-padrão da variável proteína total $(\mathrm{Pt}, \mathrm{g} / \mathrm{dL})$ durante e após o exercício de longa duração

\begin{tabular}{|c|c|c|c|c|c|c|c|}
\hline & Repouso & $1^{\circ}$ Anel & $2^{\circ}$ Anel & $3^{\circ}$ Anel & $4^{\circ}$ Anel & $1 \mathrm{~h}$ & $12 \mathrm{~h}$ \\
\hline $\mathrm{Pt}$ & $\begin{array}{l}7,05 \\
\pm 0,84^{\mathrm{a}}\end{array}$ & $\begin{array}{l}7,89 \\
\pm 0,72^{\mathrm{a}}\end{array}$ & $\begin{array}{l}7,82 \\
\pm 0,42^{\mathrm{a}}\end{array}$ & $\begin{array}{l}7,64 \\
\pm 0,99^{a}\end{array}$ & $\begin{array}{l}8,55 \\
\pm 2,97^{\mathrm{a}}\end{array}$ & $\begin{array}{l}7,56 \\
\pm 0,60^{\mathrm{a}}\end{array}$ & $\begin{array}{l}7,38 \\
\pm 0,55^{\text {a }}\end{array}$ \\
\hline
\end{tabular}

Letras diferentes na mesma linha indicam diferença pelo teste de Tukey $(\mathrm{P}<0,05)$.

\section{DISCUSSÃO}

Diante da escassez de trabalhos que apresentam dados de momentos sequenciais durante competições oficiais FEI, provavelmente devido à considerada invasividade ao se realizarem inúmeras coletas de sangue durante uma prova oficial, o presente estudo traz informações importantes a respeito do que ocorre no organismo dos equinos participantes de provas de enduro.
É importante ressaltar que, no dia da realização do teste de longa distância, as condições climáticas estavam desfavoráveis. Temperatura e umidade estavam elevadas, inclusive com presença de chuva em boa parte da prova, o que prejudicou substancialmente a qualidade do terreno. Sabe-se que velocidades e temperaturas crescentes, associadas a um terreno enlameado e escorregadio contribuem significativamente para a perda de íons pelo suor durante provas de 
enduro, além de aumentar o risco de injúrias no sistema locomotor (Robert et al., 2010). Já foi demonstrado que as condições climáticas exercem impacto no desempenho em provas de resistência (Ely et al., 2007), sendo as altas temperaturas o fator mais importante para reduzir a velocidade dos competidores.

Com relação ao índice de eliminação de competidores durante a prova encontrado no presente trabalho, ele está de acordo com o encontrado por Nagy et al. (2010), que acompanharam provas de enduro em nove países diferentes e constataram que apenas $46 \%$ dos animais que iniciaram uma prova de enduro conseguiram completá-la. As causas mais comuns de eliminação foram as claudicações, seguidas daquelas decorrentes de distúrbios metabólicos $(69,2 \%$ e $23,5 \%$, respectivamente). Os autores também verificaram que o risco de eliminação por claudicações não foi atribuído a velocidades mais rápidas, mas sim às condições do piso do percurso e a fatores ambientais.

Os aumentos nos valores de hemoglobina e hematócrito estão de acordo com o encontrado por Dumont et al. (2012) e Robert et al. (2010), que avaliaram enduros de 90 e $120 \mathrm{~km}$, respectivamente. Esse fato se deve a dois fatores principais. O primeiro deles é a esplenocontração. A mobilização da reserva esplênica é ativada em resposta a um estímulo simpático ou ao aumento da concentração de adrenalina circulante, que sensibiliza receptores adrenérgicos $\alpha-1 \mathrm{e}$, assim, promove a contração da musculatura lisa do baço. Porém, ela apresenta um efeito hemodinâmico adverso, uma vez que aumenta a viscosidade sanguínea, fazendo com que o coração tenha que aumentar a força de contração para manter o fluxo sanguíneo e a oxigenação dos tecidos (Harkins et al., 1993). Entretanto, no presente estudo, acredita-se que os aumentos observados nos valores de hematócrito devem estar mais intimamente relacionados às perdas hídricas que ocorreram, visto que a desidratação é o fator de maior influência no hematócrito em exercícios prolongados (Kingston et al, 1999).

Também associado às perdas hídricas, houve aumento nos valores numéricos de proteína total, embora esses não tenham diferido estatisticamente do basal, diferentemente do que foi encontrado por Dumont et al. (2012), que observaram aumento antes e depois de uma prova de $90 \mathrm{~km}$, e Robert et al. (2010), em cujo estudo o aumento foi significativo em prova da categoria CEI**.

Houve aumento nos valores do $\mathrm{pH}$ ao longo da prova, porém retorno aos valores basais 15 minutos após o término no exercício. Resultados semelhantes foram encontrados por Di Filippo et al. (2009) em enduro de $60 \mathrm{~km}$, os quais atribuíram esse aumento às elevadas perdas de íon cloreto, com consequente retenção de íon bicarbonato, o que caracteriza uma alcalose metabólica hipoclorêmica. As perdas eletrolíticas foram significativas, principalmente com relação aos íons cloreto, cálcio e potássio. Devido à hipertonicidade do suor equino, a diminuição nas concentrações sanguíneas dos íons sódio, potássio, cloro e cálcio é geralmente descrita ao final de provas de longas distâncias se esses valores forem comparados aos valores basais. (Robert et al., 2010; Aguilera-Tejero et al., 2001). O ânion cloreto é o íon que mais sofre perdas por meio do suor, o que explica a diminuição nos valores encontrados ao longo do exercício no presente estudo, corroborando o encontrado por Robert et al. (2010), em prova de $120 \mathrm{~km}$, Viu et al. (2010), que também avaliaram enduro de $120 \mathrm{~km}$, e Dumont et al. (2012), em prova de $90 \mathrm{~km}$. Como a perda de cloreto é elevada, no intuito de manter a eletroneutralidade do meio, o organismo mobiliza bicarbonato, por também ser um ânion, mediante mecanismos renais (McCutcheon e Geor, 1998).

Dumont et al. (2012) não encontraram diferenças no $\mathrm{pH}$ em provas de enduro, porém esse foi avaliado apenas antes e após o término da prova e os animais receberam suplementação eletrolítica. Em contrapartida, Viu et al. (2010) avaliaram uma prova de enduro de $120 \mathrm{~km} \mathrm{e}$ encontraram decréscimo nos valores de $\mathrm{pH}$, que foram associados à moderada alcalose metabólica hipoclorêmica compensada por uma acidose respiratória compensatória e acidose láctica. No presente estudo, o aumento do $\mathrm{pH}$ foi associado a reduções na $\mathrm{pCO}_{2}$, aumentos na $\mathrm{pO}_{2}$, diminuição nas concentrações de íon cloreto e aumento nos valores de $\mathrm{HCO}_{3}^{-}$. $\mathrm{O}$ aumento na $\mathrm{pO}_{2}$ e a diminuição na $\mathrm{pCO}_{2}$ indicam que houve hiperventilação, provocando aumento do volume corrente e da frequência respiratória, com o intuito de possibilitar o aumento do fluxo de $\mathrm{O}_{2}$ para os tecidos, e concomitante remoção de $\mathrm{CO}_{2}$, 
resultando em alcalose respiratória (Hopkins et al., 1998). Portanto, no presente estudo, houve distúrbio metabólico misto, caracterizado por alcalose metabólica hipoclorêmica somada à alcalose respiratória. $\mathrm{O}$ aumento da $\mathrm{pCO}_{2} 15$ minutos após o término do exercício pode ter favorecido o retorno do $\mathrm{pH}$ aos valores basais logo no início da recuperação dos animais.

Foi observada diminuição na concentração de sódio ao término do enduro até $12 \mathrm{~h}$ de recuperação, corroborando substancialmente o que foi descrito por Robert et al. (2010), porém tais resultados não foram consistentes com o encontrado por Di Filippo et al. (2009), que observaram aumento nesse íon e atribuíram esse fato à perda de fluidos circulantes. As perdas de sódio acompanham as perdas hídricas (Carlson, 1987). Diminuições nas concentrações de sódio podem corresponder à ingestão excessiva de água, o que ocasiona diluição do íon (Carlson, 1987; Hess, 2005). Considerando que os animais deste trabalho não receberam reposição eletrolítica durante o percurso, porém tiveram acesso à água ad libitum, acredita-se que a perda de sódio por meio da sudação foi a responsável pela hiponatremia, a qual se agravou com a ingestão de água.

Com relação ao cátion potássio, foi observada diminuição na sua concentração ao longo da prova. Reduções nos valores do íon potássio em exercícios prolongados ou de alta intensidade são descritas com frequência na literatura (Di Filippo et al., 2009; Ferraz et al., 2010a). A sudorese, que é bastante significativa em exercícios prolongados, aumenta a perda de potássio através do suor (McKeever, 2004). Além disso, perdas hídricas e de sódio, que também foram observadas no presente trabalho, resultam em aumento da secreção de aldosterona, que aumenta a reabsorção renal de sódio e a excreção de potássio pela urina, colaborando com a redução nos seus valores plasmáticos. Mesmo após o término do exercício, os valores de potássio continuam diminuindo porque $o$ potássio se move rapidamente para o interior das células musculares (Hess et al., 2005).

Em relação ao cálcio ionizável, houve diminuição desse íon ao longo da prova, corroborando o resultado encontrado por $\mathrm{Di}$ Filippo et al. (2009) e Dumont et al. (2012). Tal fato pode ser atribuído à perda desse íon pelo suor ou à sua migração para o meio intracelular, o que permite uma eficiente contração muscular (McKeever, 2004). A diminuição excessiva na concentração desse íon pode causar flutter diafragmático, culminando com a eliminação da competição de animais acometidos (Santos et al., 2006).

No presente estudo, não foi oferecido nenhum tipo de suplementação eletrolítica aos animais; os íons cálcio, potássio e sódio não retornaram aos valores basais, mesmo transcorridas 12 horas do término do exercício. Devido às perdas que ocorrem ao longo de provas de enduro, muitas vezes a reposição eletrolítica é recomendada. Porém, essa recomendação ainda é bastante controversa. Lacerda-Neto et al. (2003) não encontraram benefícios na reposição eletrolítica no desempenho dos animais em prova de $30 \mathrm{~km}$ de distância. Em contrapartida, Teixeira-Neto et al. (2004) sugeriram que a suplementação eletrolítica em provas de longa duração pode contribuir para o desempenho dos animais em exercícios prolongados.

Mesmo o exercício tendo sido longo e extenuante, a glicose manteve-se constante. Isso ocorre porque o exercício representa um estímulo estressante para o animal, pois ameaça a homeostase do organismo. Ocorre, então, a sensibilização do eixo hipotálamo-hipófiseadrenal, com consequente liberação de cortisol, que irá inibir a utilização de glicose pelos tecidos e estimular a gliconeogênese, fazendo com que a glicemia permaneça constante (Ferraz et al., 2010b).

Durante o exercício, houve aumento nas concentrações de lactato em relação aos valores basais, mas, apesar de esse aumento ser significativo, seus valores foram sempre inferiores a $2 \mathrm{mmol} / \mathrm{L}$, o que indica prevalência do metabolismo aeróbio. Além disso, concentrações constantes de lactato indicam que o organismo encontra-se num estado de equilíbrio estacionário dinâmico, ou seja, que a produção de lactato não supera sua utilização e eliminação (Botteon, 2012), fato extremamente desejável para cavalos de enduro. Após uma hora do término do teste, as concentrações de lactato já não diferiram dos valores basais, resultado que demonstrou bom índice de recuperação e, comprovou suficiente adaptação metabólica induzida pelo treinamento. 


\section{CONCLUSÃO}

Diante das alterações encontradas, pode-se dizer que os equinos submetidos ao exercício de enduro de $80 \mathrm{~km}$ apresentaram quadro de alcalose metabólica mista durante o exercício, por meio do desenvolvimento de alcalose metabólica hipoclorêmica e alcalose respiratória, mas essa condição foi revertida no período de recuperação. Houve predomínio do metabolismo aeróbico durante todo o exercício, como é esperado para essa modalidade. Os mecanismos de fornecimento de energia foram eficazes em manter os níveis de glicose constantes. O treinamento realizado foi efetivo em evitar a ocorrência de distúrbios metabólicos mais severos.

\section{AGRADECIMENTOS}

À Fundação de Amparo à Pesquisa do Estado de São Paulo (Fapesp), que financiou integralmente a presente pesquisa (Auxílio pesquisa $\mathrm{n}^{\circ}$ 10/09161-9 e bolsa concedida $\mathrm{n}^{\mathrm{o}}$ 2010/14294-8).

\section{REFERÊNCIAS}

AGUILERA-TEJERO, E.; ESTEPA, J.C.; LÓPEZ, I. et al. Plasma ionized calcium and parathyroid hormone concentrations in horses after endurance rides. J. Am. Vet. Med. Assoc., v.219, p.488-490, 2001.

BOTTEON, P.T L. Lactato na medicina veterinária - atualização conceitual. Rev. Bras. Med. Vet., v.34, p.283-287, 2012

CARLSON, G.P. Hematology and body fluids in the equine athlete: a review. In: GILLESPIE, J. R.; ROBINSON, N.E. Equine exercise physiology. 2.ed. Davis: ICEEP Publications, 1987. p.393-425.

CONSTABLE, P.D. A simplified strong ion model for acid-base equilibrium: applicationto horse plasma. J. Appl. Physiol., v.83, p.297-311, 1997.

DI FILIPPO, P.A.; GOMIDE, L.M.W.; OROZCO, C.A.G. et al. Alterações hemogasométricas e eletrolíticas de cavalos da raça árabe durante prova de enduro de $60 \mathrm{~km}$. Ciênc. Anim. Bras., v.10, p.840-846, 2009.
DUMONT, C.B.S.; LEITE, C.R.; MORAES, J.M. et al. Osmolaridade, ânion gap, potencial hidrogeniônico e íons plasmáticos mensuráveis de equinos Puro Sangue Árabe inalistas em provas de enduro de $90 \mathrm{~km}$. Pesqui. Vet. Bras., v.32, p.542-546, 2012.

ELY, M.R.; CHEUVRONT, S.N.; ROBERTS, W.O.; MONTAIN, S.J. Impact of weather on marathon-running performance. Med. Sci. Sport. Exerc., v.39, p.487-493, 2007

ENDURANCE Rules. 9.ed. Lausanne: Fédération Equestre Internationale, 2016. 49p. Available in: <inside.fei.org/sites/default/files/Endurance $\% 20$ Rules_2016\%20clean_0.pdf $>$. Accessed in: 28 Aug. 2016.

FERRAZ, G.C.; SOARES, O.A.B.; FOZ, N.S.B. et al. The workload and plasma ion concentration in a training match session of high-goal (elite) polo ponies. Equine Vet. J., v.42, Suppl.38, p.191-195, 2010a.

FERRAZ, G.C.; TEIXEIRA NETO, A.R.; PEREIRA, M.C. et al. Influência do treinamento aeróbio sobre o cortisol e glicose plasmáticos em equinos. Arq. Bras. Med. Vet. Zootec, v.62, p.2329, $2010 \mathrm{~b}$.

GOMIDE, L.M.W.; MARTINS, C.B.; OROZCO, C.A. et al. Concentrações sangüíneas de lactato em eqüinos durante a prova de fundo do concurso completo de equitação. Cienc. Rural, v.36, p.509-513, 2006.

HARKINS, J.D.; BEADLE, R.E.; KAMERLING, S.G. The correlation of running ability and physiological variables in Thoroughbred racehorses, Equine Vet. J., v.25, p.53-60, 1993.

HESS, T.M.; KRONFELD, D.S.; WILLIAMS, C.A. et al. Effects of oral potassium supplementation on acid-base status and plasma ion concentrations of horses during endurance exercise Am. J. Vet. Res., v.66, p.466-473, 2005.

HOPKINS, S.R.; BAYLY, W.M.; SLOCOMBE, R.F. et al. Effect of prolonged heavy exercise on pulmonary gas exchange in horses. J. Appl. Physiol., v.84, p.1723-1730, 1998. 
KINGSTON, J.K.; MCCUTCHEON, L.J.; GEOR, R.J. Comparison of three methods for estimation of exercise-related ion losses in sweat of horses. Am. J. Vet. Res., v.60, p.1248-1254, 1999.

LACERDA NETO, J.C.; SAMPAIO, R.C.L.; FERRAZ, G.C. et al. Efeitos do resfriamento intermitente e de repositor eletrolítico sobre a osmolalidade e eletrólitos séricos de eqüinos submetidos a exercício de baixa intensidade. Rev. Port. Cienc. Vet., v.98, p.189-195, 2003.

LINDINGER, M.I. Acid-base physiology during exercise and in response to training. In: HINCHCLIFF, K.W.; KANEPS, A.J.; GEOR, R.J. (Eds.). Equine sports medicine and surgery. Philadelphia: Saunders, p.872-897, 2004.

McCUTCHEON, L.J.; GEOR, R.J. Sweating. Fluid and ion losses and replacement. Vet. Clin. N. Am. Equine. Pract., v.14, p.75-95, 1998.

MCKEEVER, K.H. Body fluid and electrolytes: responses to exercise and training. In: HINCHCLIFF, K.W.; KANEPS, A.J.; GEOR, R.J. (Eds.). Equine sports medicine and surgery. Basic and clinical sciences of equine athlete. Philadelphia: Saunders Press, 2004. p.854-871.

NAGY, A.; DYSON, S.J.; MURRAY. J.K. A veterinary review of endurance riding as an international competitive sport. Vet. J., v.194, p.288-293, 2012.

NAGY, A.; MURRAY, J.; DYSON, S. Elimination from elite endurance rides in nine countries - a preliminary study. Equine Vet. J., Suppl.42, p.637-643, 2010.
OLIVEIRA, J.C.; BALDISSERA, V.; SIMÕES, H.G. et al. Identificação do limiar de lactato e limiar glicêmico em exercícios resistidos. Rev. Bras. Med. Esporte, v.12, p.333-338, 2006.

ROBERT, C.; GOACHET, A.G.; FRAIPONT, A. et al. Hydration and electrolyte balance in horses during an endurance season. Equine Vet. J., Suppl.38, p.98-104, 2010.

SANTOS, S.A.; SILVA, R.A.M.S.; AZEVEDO, J.R.M. et al. Serum electrolyte and total protein alterations in Pantaneiro horse during long distance exercise. Arq. Bras. Med. Vet. Zootec., v.53, p.351-357, 2006.

SCHOTT II, H.C.; HINCHCLIFF, K.W. Fluids, electrolytes, and bicarbonate. Vet. Clin. N. Am. Equine. Pract., v.9, p.577-604, 1993.

SILVA, M.A.G.; MARTINS, C.B.; GOMIDE, L.M.W. et al. Determinação de eletrólitos, gases sanguíneos, osmolalidade, hematócrito, hemoglobina, base titulável e anion gap no sangue venoso de equinos destreinados submetidos a exercício máximo e submáximo em esteira rolante. Arq. Bras. Med. Vet. Zootec., v.61, n.5, 2009.

TEIXEIRA NETO, A.R.; FERRAZ, G.C.; MATAQUEIRO, M.I. et al. Reposição eletrolítica sobre variáveis fisiológicas de cavalos em provas de enduro de 30 e $60 \mathrm{Km}$. Cien. Rural, v.34, p.1505-1511, 2004.

VIU, J.; JOSE-CUNILLERAS, E.; ARMENGOU, L. et al. Acid-base imbalances during a $120 \mathrm{~km}$ endurance race compared by traditional and simplified strong ion difference methods. Equine Vet. J., v.42, Suppl.38, p.76-82, 2010. 